\title{
SCALE DEPENDENCE OF DARK ENERGY ANTIGRAVITY
}

\author{
L. Perivolaropoulos* \\ Institute of Nuclear Physics, \\ National Centre for Scientific Research "Demokritos N.C.S.R.", \\ Athens, Greece \\ leandros@mail.demokritos.gr
}

\begin{abstract}
We investigate the effects of negative pressure induced by dark energy (cosmological constant or quintessence) on the dynamics at various astrophysical scales. Negative pressure induces a repulsive term (antigravity) in Newton's law which dominates on large scales. Assuming a value of the cosmological constant consistent with the recent SnIa data we determine the critical scale $r_{c}$ beyond which antigravity dominates the dynamics $\left(r_{c} \sim 1 M p c\right)$ and discuss some of the dynamical effects implied. We show that dynamically induced mass estimates on the scale of the Local Group and beyond are significantly modified due to negative pressure. We also briefly discuss possible dynamical tests (eg effects on local Hubble flow) that can be applied on relatively small scales (a few $M p c)$ to determine the density and equation of state of dark energy.
\end{abstract}

Keywords: Cosmological Constant, Newton's law, Dark Energy, Galactic Dynamics, Hubble Flow

\section{Introduction}

Recent cosmological observations extending the Hubble diagram of high redshift Type Ia supernonovae performed independetly by two groups (the Supernova Cosmology Project [2] and the High-Z Supernova Team $[3,4]$ presented evidence that the expansion of the universe is accelerating rather than slowing down. This fact combined with the Boomerang and Maxima-1 measurements of the first acoustic peak location in the angular power spectrum of the cosmic microwave background (CMB) $[5,6]$ point towards a standard cosmological model with critical density

* Contributed talk at the 2nd Hellenic Cosmology Workshop at NOA (Athens) Jan. 2001. Based on work done in collaboration with M. Axenides and E. Floratos[1] 
$\left(\Omega=\Omega_{m}+\Omega_{\Lambda}=1\right)$ and a dominant $\Lambda$-like, "dark energy" component at the present epoch $\left(\Omega_{\Lambda} \approx 0.7\right)$. This component could be produced by non-zero and positive cosmological constant $\Lambda$ with

$$
\Lambda \simeq 10^{-52} m^{-2}
$$

Such a term can produce the required repulsive force to explain the accelerating universe phenomenon. A diverse set of other cosmological observations also compellingly suggest that the universe posesses a nonzero negative pressure component corresponding to vacuum energy density of the same order as the matter energy density[7, 8, 9].

In addition to causing an acceleration to the expansion of the universe the existence of a non-zero cosmological constant would have interesting gravitational effects on various astrophysical scales[10, 1]. For example it would affect gravitational lensing statistics of extragalactic surveys [11], large scale velocity flows[12] and there have been some claims that even smaller systems (galactic[13] and planetary[14]) could be affected in an observable way by the presence of a cosmological constant consistent with cosmological expectations. Even though some of these claims were falsified $[15,16,17]$ the scale dependence of the dynamical effects of vacuum energy remains an interesting open issue.

The effects of the vacuum energy on cosmological scales and on local dynamics can be obtained from the Einstein equations which in the presence of a non-zero cosmological constant are written as

$$
R_{\mu \nu}-\frac{1}{2} g_{\mu \nu} R=8 \pi G T_{\mu \nu}
$$

These equations, under the assumptions of spherical symmetric energymomentum (EM) tensor $T_{k}^{i}=\rho_{Q} c^{2} \operatorname{diag}(1,-w,-w,-w)$ and a mixture of dust-like matter $\left(\rho=\rho_{m}, w=0\right)$ and dark energy $\left(\rho=\rho_{Q}\right.$, $-\frac{1}{3} \leq w \leq-1$ ) lead (for the $1-1$ component) to the generalized Newton's equation

$$
\ddot{r}=-G M_{e f f} / r^{2} ; \quad M_{e f f}=M_{m}(r)+M_{Q}(r) .
$$

where $M(r)=\frac{4 \pi}{3}(1+3 w) \rho r^{3}$. Notice that for $w<-\frac{1}{3}$ we have negative gravitating effective mass (antigravity) which can lead to accelerated cosmological expansion and to non-trivial dynamical effects on astrophysical scales. The accelerated cosmological expansion is obtained for $w<-\frac{1}{3}$ from the Friedman equations which for $k=0$ imply

$$
\begin{aligned}
\rho_{Q} & \sim R_{Q}^{-3(1+w)} \\
R_{Q} & \sim t^{\frac{2}{3(1+w)}}
\end{aligned}
$$


where $R_{Q}$ is the scale factor of the universe. In what follows we focus on the effects of dark energy with $w=-1$ (cosmological cosntant). The more general case of $-1<w \leq-\frac{1}{3}$ (quintessence[18]) will be discussed elsewhere.

The vacuum energy implied from eq. (1) $\left(10^{-10} \mathrm{erg} / \mathrm{cm}^{3}\right)$ is less by many orders of magnitude than any sensible estimate based on particle physics. In addition, the matter density $\rho_{m}$ and and the vacuum energy $\rho_{\Lambda}$ evolve at different rates, with $\rho_{m} / \rho_{\Lambda} \simeq R^{-3}$ and it would seem quite unlikely that they would differ today by a factor of order unity. Interesting attempts have been made during the past few years to justify this apparent fine tuning by incorporating evolving scalar fields (quintessence [18]) or probabilistic arguments based on the anthropic principle[19]).

For $w=-1$ we have Einstein's cosmological constant with $\Lambda=$ $8 \pi G \rho_{\Lambda} / c^{2}$ (cosmological vacuum with $w=-1$ ) and the gravitating mass is $M_{\Lambda}=-8 \pi \rho_{\Lambda} r^{3} / 3$. Thus the generalized Newtonian potential leads to a gravitational interaction acceleration

$$
\ddot{r}=-\frac{G M}{r^{2}}+\frac{\Lambda c^{2}}{3} r
$$

This generalized force includes a repulsive term

$$
g_{r}=\frac{\Lambda c^{2}}{3} r
$$

which is expected to dominate at distances larger than

$$
r_{c}=\left(\frac{3 G M}{\Lambda c^{2}}\right)^{\frac{1}{3}} \simeq 10^{2}\left(\frac{\bar{M}_{1}}{\bar{\Lambda}_{52}}\right)^{\frac{1}{3}} p c \simeq 2 \times 10^{7}\left(\frac{\bar{M}_{1}}{\bar{\Lambda}_{52}}\right)^{\frac{1}{3}} A U
$$

where $\bar{M}_{1}$ is the mass within a sphere of radius $r_{c}$ in units of solar masses $M_{\odot}=2 \times 10^{30} \mathrm{~kg}$ and $\bar{\Lambda}_{52}$ is the cosmological constant in units of $10^{-52} \mathrm{~m}^{-2}$.

The question we address in this report is the following: 'What are the effects of the additional repulsive force $g_{r}$ on the various astrophysical scales?' This issue has been addressed in the literature for particular scales. For example it was shown[15] that the effects of this term in the solar system could only become measurable (by modifying the perihelia precession) if the cosmological constant were fourteen orders of magnitude larger than the value implied by the SnIa observations.

In the next section it will be shown that the vacuum energy required to close the universe (eq. (1)) has negligible effects on the dynamics of galactic scales (few tens of $\mathrm{kpc}$ ). The dynamically derived mass to light ratios of galaxies obtained from velocity measurements on galactic scales are modified by less than $0.1 \%$ due to the vacuum energy term of eq. (1). 
This is not true however on cluster scales or larger. Even on the scales of the Local Group of galaxies (about $1 \mathrm{Mpc}$ ) the gravitational effects of the vacuum energy are significant. We show that the dynamically obtained masses of M31 and the Milky Way must be increased by about 35\% to compensate the repulsion of the vacuum energy of eq. (7) and produce the observed relative velocity of the members of the Local Group. The effects of vacuum energy are even more important on larger scales (rich cluster and supercluster).

\section{Scale Dependence of Antigravity}

In order to obtain a feeling of the relative importance of antigravity vs gravity on the various astrophysical scales it is convenient to consider the ratio of the corresponding two terms in eq. (6). This ratio $q$ may be written as

$$
q=\frac{\Lambda c^{2} r^{3}}{3 G M} \simeq 0.5 \times 10^{-5} \frac{\bar{\Lambda}_{52} \bar{r}_{1}^{3}}{\bar{M}_{1}}
$$

where $\bar{r}_{1}$ is the distance measured in units of $p c$. For the solar system ( $\bar{r}_{1} \simeq 10^{-5}, \bar{M}_{1}=1$ ) we have $q_{s s} \simeq 10^{-20}$ which justifies the fact that interplanetary measures can not give any useful bound on the cosmological constant.

For a galactic system $\left(\bar{r}_{1} \simeq 10^{4}, \bar{M}_{1}=10^{10}\right)$ we have $q_{g} \simeq 5 \times 10^{-4}$ which indicates that up to galactic scales the dynamical effects of the antigravity induced by $\Lambda$ are negligible. On a cluster however $\left(\bar{r}_{1} \simeq 10^{7}\right.$, $\bar{M}_{1}=10^{14}$ ) we obtain $q_{c} \simeq O(1)$ and the gravitational effects of the vacuum energy become significant. This will be demonstrated in a more quantitative way in what follows.

The precessions of the perihelia of the planets provide one of the most sensitive Solar System tests for the cosmological constant. The additional precession due to the cosmological constant can be shown[15] to be

$$
\Delta \phi_{\Lambda}=6 \pi q \mathrm{rad} / \mathrm{orbit}
$$

where $q$ is given by eq. (9). For Mercury we have $\bar{r}_{1} \simeq 10^{-6}$ which leads to $q_{m c} \simeq 10^{-23}$ and $\Delta \phi_{\Lambda} \simeq 10^{-22} \mathrm{rad} /$ orbit. The uncertainty in the observed precession of the perihelion of Mercury is $0.1^{\prime \prime}$ per century or $\Delta \phi_{\text {unc }} \simeq 10^{-9} \mathrm{rad} /$ orbit which is 13 orders of magnitude larger than the one required for the detection of a cosmologicaly interesting value for the cosmological constant. The precession per century ${ }^{1}$ scales like $\bar{r}_{1}^{3 / 2}$ and therefore the predicted additional precession per century for distant planets $\left(\bar{r}_{1}(\right.$ Pluto $) \simeq 10^{2} \bar{r}_{1}$ (Mercury) $)$ due to the cosmological constant increases by up to 3 orders of magnitude. It remains however approximatelly 10 orders of magnitude smaller than the precession re- 
quired to give a cosmologically interesting detection of the cosmological constant even with the best quality of presently available observations. It is therefore clear that since the relative importance of the gravitational contribution is inversely proportional to the mean matter density on the scale considered, a cosmological constant could only have detectable gravitational effects on scales much larger than the scale of the solar system.

On galactic scales, the rotation velocities of spiral galaxies as measured in the $21 \mathrm{~cm}$ line of neutral hydrogen comprise a good set of data for identifying the role of the vacuum energy. This is because these velocity fields usually extend well beyond the optical image of the galaxy on scales where the effects of $\Lambda$ are maximized and because gas on very nearly circular orbits is a precise probe of the radial force law. For a stable circular orbit with velocity $v_{c}$ at a distance $r$ from the center of a galaxy with mass $M$ we obtain using eq. (6)

$$
v_{c}^{2}=\frac{G M}{r}-\frac{\Lambda c^{2} r^{2}}{3}
$$

We now define the rescaled dimensionless quantities $\bar{v}_{100}, \bar{r}_{10}$ and $\bar{M}_{10}$ as follows:

$$
\begin{aligned}
v_{c} & =100 \bar{v}_{100} \mathrm{~km} / \mathrm{sec} \\
r & =10 \bar{r}_{10} \mathrm{kpc} \\
M & =10^{10} \bar{M}_{10} M_{\odot}
\end{aligned}
$$

Eq. (11) may now be written in a rescaled form as

$$
\bar{v}_{100}^{2}=\frac{1}{2} \frac{\bar{M}_{10}}{\bar{r}_{10}}-3 \times 10^{-5} \bar{\Lambda}_{52} \bar{r}_{10}^{2}
$$

In order to calculate the effects of the cosmological constant on the dynamically obtained masses of galaxies (including their halos) it is convenient to calculate the ratio

$$
p \equiv \frac{M\left(\bar{\Lambda}_{52}=1\right)-M\left(\bar{\Lambda}_{52}=0\right)}{M\left(\bar{\Lambda}_{52}=0\right)}=\frac{3 \times 10^{-5} \bar{r}_{10}^{2}}{\bar{v}_{100}^{2}}
$$

where $M\left(\Lambda_{52}\right)$ is the dynamically calculated mass obtained from equation (13). In Table 1 we show a calculation of the mass ratio $p$ for 22 galaxies of different sizes and rotation velocities[20]. The corresponding plot of $p(r)$ is shown in Fig. 1.

It is clear that even for large galaxies where the role of the repulsive force induced by vacuum energy is maximized, the increase of the mass needed to compensate vacuum energy antigravity is negligible. In order 


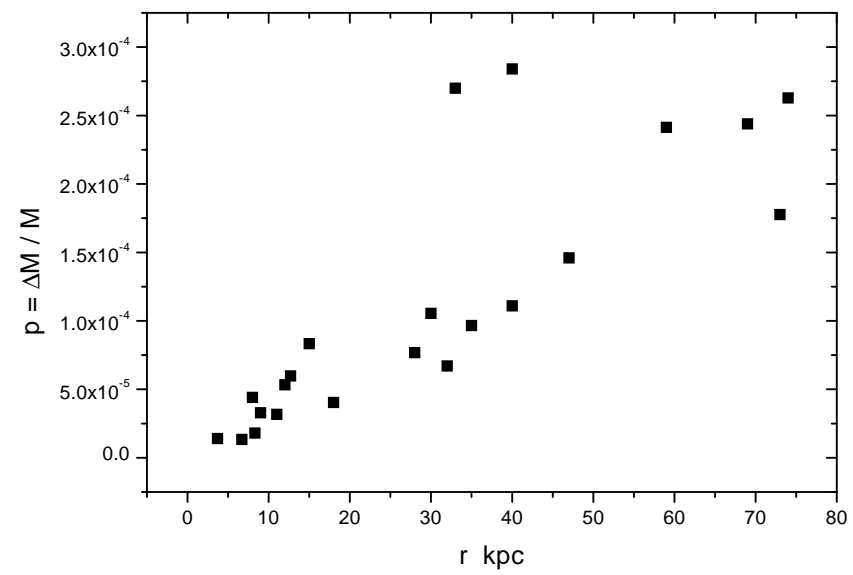

Figure 1. Relative increase $p$ of dynamically calculated mass of galaxies due to repusive effects vacuum energy vs galaxy radious $r$ measured in kpc.

for these effects to be significant the cosmological constant would have to be larger that the value required for flatness by a factor of at least $10^{3}$.

Such value would be inconsistent with several cosmological observations. Therefore, even though the effects of vacuum energy on galactic dynamics are much more important compared to the corresponding effects on solar system dynamics it is clear that we must consider systems on even larger scales where the mean density is smaller in order to obtain any nontrivial effects on the dynamics.

The Local Group of galaxies is a particularly useful system for studying mass dynamics on large scales because it is close enough to be measured and modeled in detail yet it is large enough (and poor enough) to probe the effects of vacuum energy on the dynamics. The dominant members of the group are the Milky Way and the Andromeda Nebula M31. Their separation is

$$
r_{0} \equiv r\left(t=t_{0}\right) \simeq 800 k p c
$$

and the rate of change of their separation is

$$
\frac{d r}{d t}\left(t=t_{0}\right) \simeq-123 k m s^{-1}
$$


Table 1. Relative increase $p$ of dynamically calculated mass of galaxies due to repulsive effects of vacuum energy.

\begin{tabular}{lccc}
\hline Galaxy & $r_{H I} k p c$ & $v_{\text {rot }} k m / s e c$ & $p$ \\
\hline UGC2885 & 73 & 300 & $17.8 \times 10^{-5}$ \\
NGC5533 & 74 & 250 & $26.3 \times 10^{-5}$ \\
NGC6674 & 69 & 242 & $24.3 \times 10^{-5}$ \\
NGC5907 & 214 & $6.7 \times 10^{-5}$ \\
NGC2998 & 32 & 213 & $14.6 \times 10^{-5}$ \\
NGC801 & 47 & 208 & $24.1 \times 10^{-5}$ \\
NGC5371 & 59 & 208 & $11.1 \times 10^{-5}$ \\
NGC5033 & 40 & 195 & $9.7 \times 10^{-5}$ \\
NGC3521 & 175 & $7.7 \times 10^{-5}$ \\
NGC2683 & 35 & 155 & $4.0 \times 10^{-5}$ \\
NGC6946 & 28 & 160 & $10.5 \times 10^{-5}$ \\
UGC128 & 18 & 130 & $28.4 \times 10^{-5}$ \\
NGC1003 & 30 & 107 & $27.0 \times 10^{-5}$ \\
NGC247 & 40 & 107 & $3.1 \times 10^{-5}$ \\
M33 & 33 & 100 & $1.8 \times 10^{-5}$ \\
NGC7793 & 11 & 90 & $1.3 \times 10^{-5}$ \\
NGC300 & 8.3 & 90 & $6.0 \times 10^{-5}$ \\
NGC5585 & 6.7 & 90 & $5.3 \times 10^{-5}$ \\
NGC2915 & 12.7 & 86 & $8.3 \times 10^{-5}$ \\
NGC55 & 12 & 66 & $3.2 \times 10^{-5}$ \\
IC2574 & 15 & 54 & $4.4 \times 10^{-5}$ \\
DDO168 & 9 & & $1.4 \times 10^{-5}$ \\
\hline & 8 & &
\end{tabular}

A widely used assumption is that the motion of approach of M31 and Milky Way is due to the mutual gravitational attraction of the masses of the two galaxies. Adopting the simplest model of the Local Group as an isolated two body system, the Milky Way and M31 have negligible relative angular momentum and their initial rate of change of separation is zero in comoving coordinates. The equation of motion for the separation $r(t)$ of the centers of the two galaxies in the presence of a nonzero cosmological constant is:

$$
\frac{d^{2} r}{d t^{2}}=-\frac{G M}{r^{2}}+\frac{\Lambda c^{2}}{3} r
$$

where $\mathrm{M}$ is the sum of the masses of the two galaxies. A similar equation (with $\Lambda=0$ ) was used in Ref [21] to obtain an approximation of the mass to light ratio of the galaxies of the Local Group. Numerical studies[22] have shown that this approximation is reasonable and leads to a relatively small overestimation (about 25\%) of the galactic masses. This correction is due to the effects of the other dwarf members of the 
Local Group that are neglected in the isolated two body approximation. Here we are not interested in the precise evaluation of the masses of the galaxies but on the effects of the cosmological constant on the evaluation of these masses. Therefore we will use the 'isolated two body approximation' of the Local Group (eq. (17)) and focus on the dependence of the calculated value of the mass $M$ as a function of $\Lambda$ in the range of cosmologicaly interesting values of $\Lambda$. Our goal is to find the total mass $M$ of the Local Group galaxies, using eq. (17) supplied with the following conditions:

$$
\begin{aligned}
r\left(t=t_{0}\right) & =800 \mathrm{kpc} \\
\frac{d r}{d t}\left(t=t_{0}\right) & =-123 \mathrm{~km} / \mathrm{sec} \\
\frac{d r}{d t}(t=0) & =0
\end{aligned}
$$

where $t_{0}=15 G y r$. Upon integrating and rescaling eq. (17) we obtain

$$
\left(\frac{d \bar{r}_{100}}{d \bar{t}_{15}}\right)^{2}=\bar{M}\left(\frac{1}{\bar{r}_{100}}-\frac{1}{8}\right)+\bar{\Lambda}\left(\bar{r}_{100}^{2}-64\right)+420 \equiv f\left(\bar{r}_{100}\right)
$$

where we have used condition (19) and the rescaled quantities defined by

$$
\begin{aligned}
r & =100 \bar{r}_{100} k p c \\
t & =1.5 \times 10^{10} \bar{t}_{15} \text { yrs } \\
M & =4 \times 10^{8} \bar{M} M_{\odot} \\
\Lambda & =1.3 \bar{\Lambda}_{52}
\end{aligned}
$$

Using now conditions (18) and (20) we obtain the equation that can be solved to evaluate the galactic masses for various $\Lambda$

$$
1=\bar{t}_{15}\left(t=t_{0}\right)=-\int_{\bar{r}_{100}(t=0)}^{\bar{r}_{100}\left(t=t_{0}\right)} \frac{d r}{\sqrt{f\left(\bar{r}_{100}\right)}}
$$

The lower limit of the integral (26) is obtained by solving condition (20) for $r$ (using eq. (21) while the upper limit is given by eq. (18) in its rescaled form. This equation can be solved numerically for $M$ to calculate the galactic total mass $M$ for various values of the cosmological constant $\Lambda$. The resulting dependence of $M$ on $\Lambda$ is shown in Fig. 2 (continuous line).

Clearly, for a value of $\Lambda$ consistent with the recent SnIa observations $\left(\Lambda \simeq 0.7 \times 10^{-52} \mathrm{~m}^{-2}\right)$ the calculated galactic masses using Local Group dynamics are $35 \%$ larger than the corresponding masses calculated with 


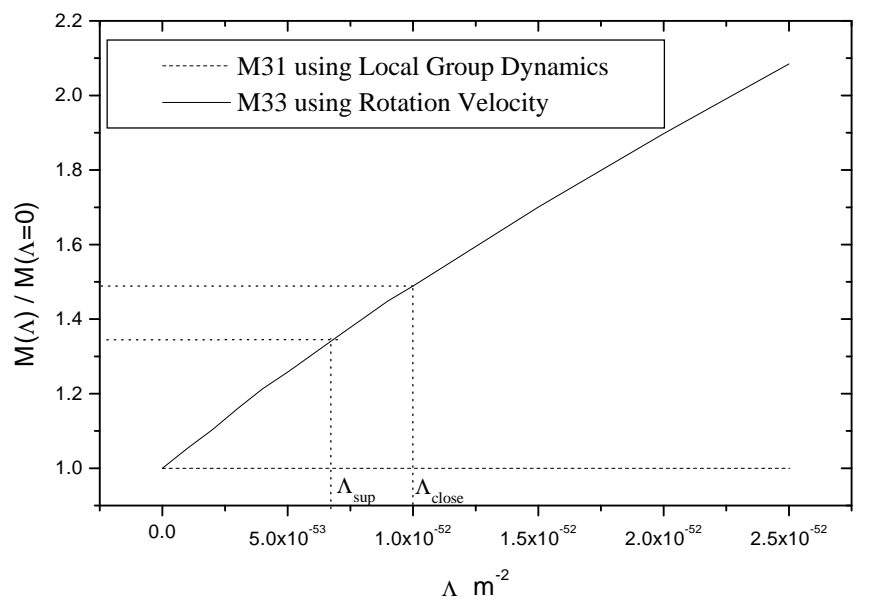

Figure 2. The relative galactic total mass $\frac{M(\Lambda)}{M(\Lambda=0)}$ calculated for various values of the cosmological constant $\Lambda$ using Local Group (continous line) and galactic scale (M33, dashed line) velocity data. The dotted lines correspond to the $\Lambda$ value implied by the SnIa data and to $\Lambda_{c l}$ for which the vacuum energy alone closes the universe.

$\Lambda=0$. On Fig. 2 we also plot (dashed line) the dependence of the $\frac{M(\Lambda)}{M(\Lambda=0)}$ on $\Lambda$ calculated using galactic dynamics (rotation velocity) of the galaxy M33. Clearly a galactic system in contrast to the Local Group is too small and dense to be a sensitive detector of the cosmological constant.

\section{Discussion}

We conclude that the Local Group of galaxies is a system that is large enough and with low enough matter density to be a sensitive probe of the gravitational effects of a cosmological constant with value consistent with cosmological expectations and recent SnIa obsrevations. Smaller and denser systems do not have this property. On the other hand the gravitational effects of $\Lambda$ should be even more pronounced on larger cosmological systems.

The fact that the dynamical effects of negative pressure are manifested on scales of about $1 M p c$ and larger opens up interesting new windows for the determination of the density and equation of state of dark energy. For example the gravitational effects discussed here can be used as an independent detection method of the cosmological constant, if the galac- 
tic masses of systems like the Local Group are measured independently using non-dynamical methods (eg gravitational lensing). In that case $\rho_{\Lambda}$ could be obtained using plots like the one shown in Fig. 2. Also the unexpected quietness and linearity of the local Hubble flow in the very clumpy local universe $(1-10 \mathrm{Mpc})$ can be attributed to the negative pressure[23] of the dark energy and used to constrain $\rho_{Q}$ and $w$.

\section{Acknowledgements}

We would like to thank M. Plionis for useful conversation.

\section{Notes}

1. The angular velocity is smaller for distant planets and therefore the precession per century does not scale like the $\bar{r}_{1}^{3}$ as the precession per orbit does

\section{References}

[1] M. Axenides, E. G. Floratos and L. Perivolaropoulos, Mod. Phys. Lett. A 15, 1541 (2000) [astro-ph/0004080].

[2] S. Perlmutter et al. [Supernova Cosmology Project Collaboration], Ap. J. 517, 565 (1999); astro-ph/9812133.

[3] B. P. Schmidt et al. [Hi-Z Supernova Team Collaboration], Astrophys. Journ. 507, 46 (1998); astro-ph/9805200;

[4] A. G. Riess et al., Astron. J. 116, 1009 (1998)[astro-ph/9805201].

[5] de Bernardis et al. Nature, 404, 955 (2000).

[6] Jaffe A.H. et al. astro-ph/0007333 (2000).

[7] S. Weinberg, "Theories of the cosmological constant," astro-ph/9610044.

[8] L. M. Krauss and M. S. Turner, Gen. Rel. Grav. 27, 1137 (1995) [astro$\mathrm{ph} / 9504003]$.

[9] S. M. Carroll, W. H. Press and E. L. Turner, Ann. Rev. Astron. Astrophys. 30, 499 (1992).

[10] J. P. Ostriker and P. J. Steinhardt, Nature 377, 600 (1995).

[11] R. Quast and P. Helbig, Astron. Astrophys. 344, 721 (1999)[astro-ph/9904174].

[12] I. Zehavi and A. Dekel, astro-ph/9904221.

[13] S. B. Whitehouse and G. V. Kraniotis, astro-ph/9911485.

[14] J. Cardona and J. Tejeiro, Ap. J. 493, 52 (1998).

[15] E. L. Wright, astro-ph/9805292.

[16] I. P. Neupane, gr-qc/9902039.

[17] M. D. Roberts, Mon. Not. Roy. Astron. Soc. 228, 401 (1987).

[18] R. R. Caldwell, R. Dave and P. J. Steinhardt, Phys. Rev. Lett. 80, 1582 (1998) [astro-ph/9708069]; I. Zlatev, L. Wang and P. J. Steinhardt, Phys. Rev. Lett. 82, 896 (1999) [astro-ph/9807002]. 
[19] J. Garriga and A. Vilenkin, J. Garriga, M. Livio and A. Vilenkin, Phys. Rev. D61, 023503 (2000)[astro-ph/9906210].

[20] R.H. Sanders, Ap. J. 473, 117 (1996).

[21] P.J.E. Peebles, Principles of Physical Cosmology, Princeton Series in Physics (1993).

[22] P.J.E. Peebles, A.L. Melott, M.R. Holmes and L.R. Jiang, Ap. J. 345, 108 (1989).

[23] Y. Baryshev, A. Chernin and P. Teerikorpi, astro-ph/0011528 